\title{
Detailed Models for Timing and Efficiency in Resistive Plate Chambers
}

\author{
Werner Riegler ${ }^{\mathrm{a}}$, Christian Lippmann ${ }^{\mathrm{a}}$

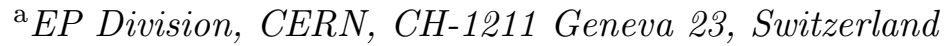

\begin{abstract}
We discuss detailed models for detector physics processes in Resistive Plate Chambers, in particular including the effect of attachment on the avalanche statistics. In addition we present analytic formulas for average charges and intrinsic RPC time resolution. Using a Monte Carlo simulation including all the steps from primary ionization to the front-end electronics we discuss the dependence of efficiency and time resolution on parameters like primary ionization, avalanche statistics and threshold.
\end{abstract}

\section{Introduction}

The two RPC geometries discussed in this report are shown in Fig. 1. 'Trigger RPCs' similar to the ones used for ATLAS [1] with a $2 \mathrm{~mm}$ gas gap and $2 \mathrm{~mm}$ Bakelite plates with a volume resistivity of $\approx 10^{10} \Omega \mathrm{cm}$ show efficiencies of $>99 \%$ and time resolution of about 1 ns. 'Timing RPCs' like the ones developed by Fonte et. al. [2] with a $300 \mu \mathrm{m}$ gas gap show efficiencies of $\approx$ $75 \%$ and time resolutions down to 50 ps. The trigger RPCs use the gas mixture $\mathrm{C}_{2} \mathrm{~F}_{4} \mathrm{H}_{2} / \mathrm{i}_{-} \mathrm{C}_{4} \mathrm{H}_{10} / \mathrm{SF}_{6} 97 / 2.5 / 0.5$ [8] and timing RPCs use the mixture $\mathrm{C}_{2} \mathrm{~F}_{4} \mathrm{H}_{2} / \mathrm{i}-\mathrm{C}_{4} \mathrm{H}_{10} / \mathrm{SF}_{6} 85 / 25 / 10$.
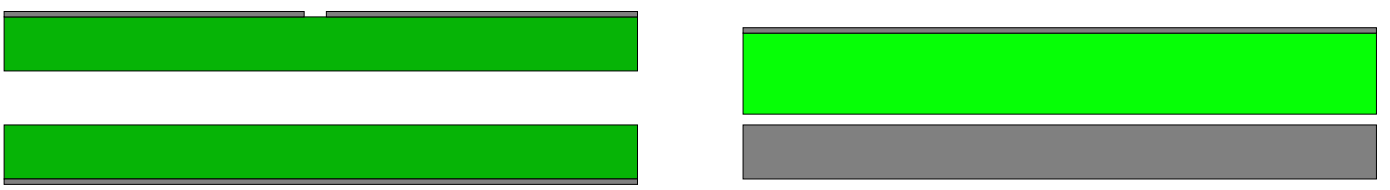

Fig. 1. RPC geometries discussed in this report. The left figure shows a trigger RPC with a gas gap of $2 \mathrm{~mm}$ and $2 \mathrm{~mm}$ Bakelite plates [1]. The right figure shows a timing $\mathrm{RPC}$ with a gas gap of $300 \mu \mathrm{m}$, a $3 \mathrm{~mm}$ glass plate and $2 \mathrm{~mm}$ aluminum plate [2] .

Preprint submitted to Elsevier Preprint 7 January 2002 


\section{Gas Transport Properties}

The primary ionization is characterized by a given number of clusters $/ \mathrm{mm}$, which is Poisson distributed, and the number of electrons per cluster, which is approximately $1 / n^{2}$ distributed. Figure 2 shows the numbers predicted by HEED [3]. At a Pion energy of $7 \mathrm{GeV}$ we expect 9-10 primary clusters $/ \mathrm{mm}$.

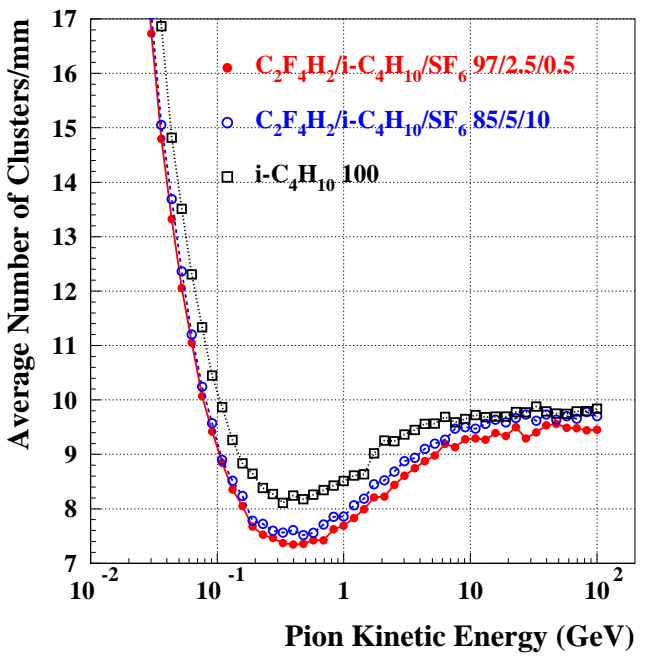

(a)

(b)

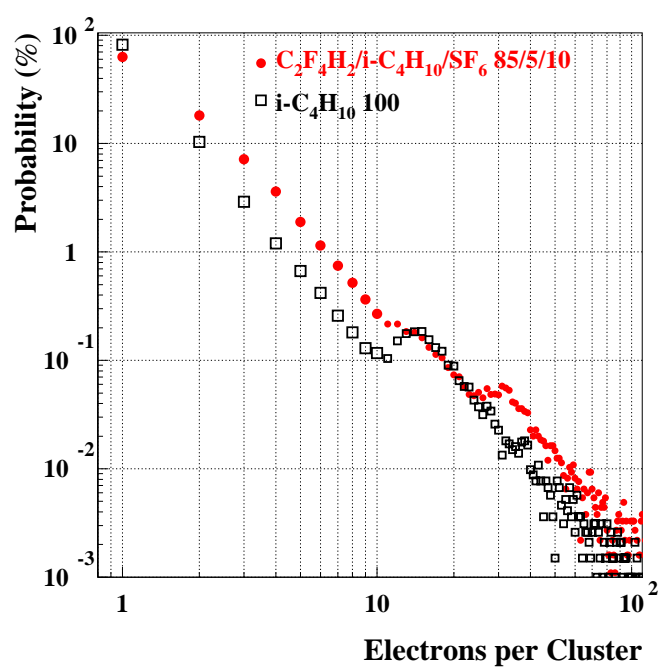

Fig. 2. Primary ionization for different gases as simulated by HEED [3]. Average number of clusters/mm (a) and cluster size distribution (b). The numbers for isobutane are shown as a reference.

The trigger RPCs operate at about $10 \mathrm{kV}$ giving a field of $50 \mathrm{kV} / \mathrm{cm}$ in the gas gap. The timing RPCs operating at $3 \mathrm{kV}$ giving a field of about $100 \mathrm{kV} / \mathrm{cm}$. The Townsend coefficient $\alpha$ and attachment coefficient $\eta$ for these voltages as prediced by IMONTE [4] is shown in Figure 3. For the trigger RPCs we expect an effective Townsend coefficient of $\approx 10 / \mathrm{mm}$ while for the timing RPCs we expect about $100 / \mathrm{mm}$. 


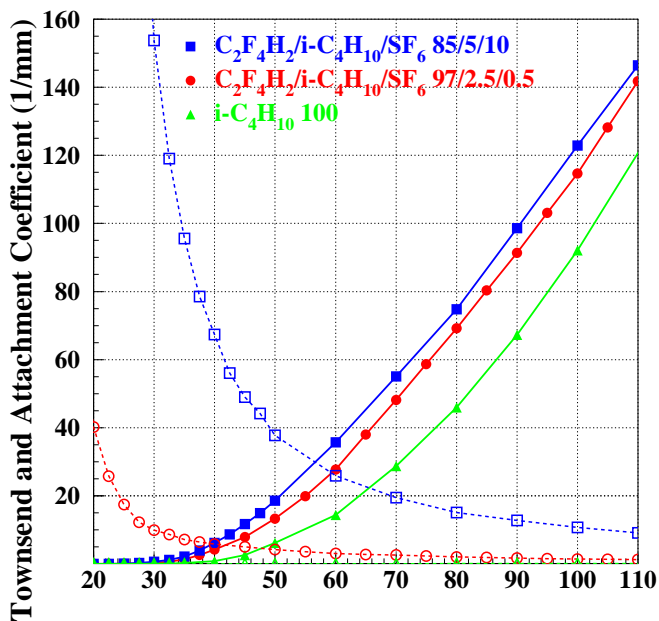

(a)

Electric Field $(\mathbf{k V} / \mathrm{cm})$

(b)

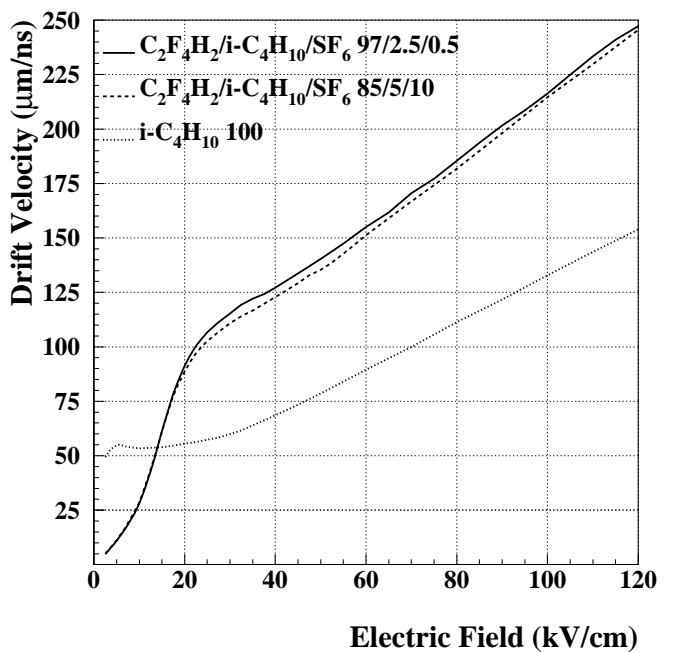

Fig. 3. (a) Townsend (solid line) and attachment (broken line) coefficient simulated with IMONTE [4]. (b) Drift velocity as simulated with MAGBOLTZ [5].

The avalanche fluctuations are included by a model from Legler [9] where the probability to find $n$ electrons after a distance $x$ for an avalanche started with one electrons at $x=0$ is given by

$$
\begin{array}{rlrl}
P(n, x) & =k \frac{\bar{n}(x)-1}{\bar{n}(x)-k} & n=0 \\
& =\bar{n}(x)\left(\frac{1-k}{\bar{n}(x)-k}\right)^{2}\left(\frac{\bar{n}(x)-1}{\bar{n}(x)-k}\right)^{n-1} & & n>0
\end{array}
$$

with

$$
\bar{n}(x)=e^{(\alpha-\eta) x} \quad k=\frac{\eta}{\alpha}
$$


Finally the drift velocity, as predicted by MAGBOLTZ [5] is also shown in Figure 3 . We expect drift velocities of $\approx 130 \mu \mathrm{m} / \mathrm{ns}$ for the trigger $\mathrm{RPC}$ and $\approx 220 \mu \mathrm{m} / \mathrm{ns}$ for the timing RPCs.

The induced current signals are given by

$$
I(t)=\frac{E_{w}}{V_{w}} e_{0} v N(t)
$$

where $N(t)$ is the number of electrons present at time $t, v$ is the electron drift velocity, $e_{0}$ is the electron charge and $E_{w} / V_{w}$ is given by

$$
\text { single gap: } \quad \frac{E_{w}}{V_{w}}=\frac{\epsilon_{r}}{2 b+d \epsilon_{r}} \quad \text { quad gap: } \quad \frac{E_{w}}{V_{w}}=\frac{\epsilon_{r}}{b+2 d \epsilon_{r}}
$$

where $d$ is the gas gap, $b$ is the Bakelite (glass) thickness and $\epsilon_{r}$ is the Bakelite (glass) relative dielectric constant [10]. All the above parameters were put into a simple Monte Carlo Program. Setting a threshold to the current signal from each event finally gives the efficiency and time resolution.

\section{$3 \quad$ Efficiency Results}

The simulated efficiency for the trigger and timing RPCs together with measurements are shown in Figure 4. The simulated numbers are not far from the measurements. We suspect the reason in the small discrepancy in the attachment coefficient which is the most uncertain number of all the detector physics parameters. Multiplying the attachment coefficient by 0.65 for the timing RPC and 0.45 for the trigger RPC gives a perfect match between simulation and measurement.

We conclude that the measured efficiencies are close to what we expect from 'standard' detector physics.

\section{Timing Results}

To get an order of magnitude estimate for the RPC time resolution we first assume the RPC signal

$$
i(t)=A e^{(\alpha-\eta) v t}
$$




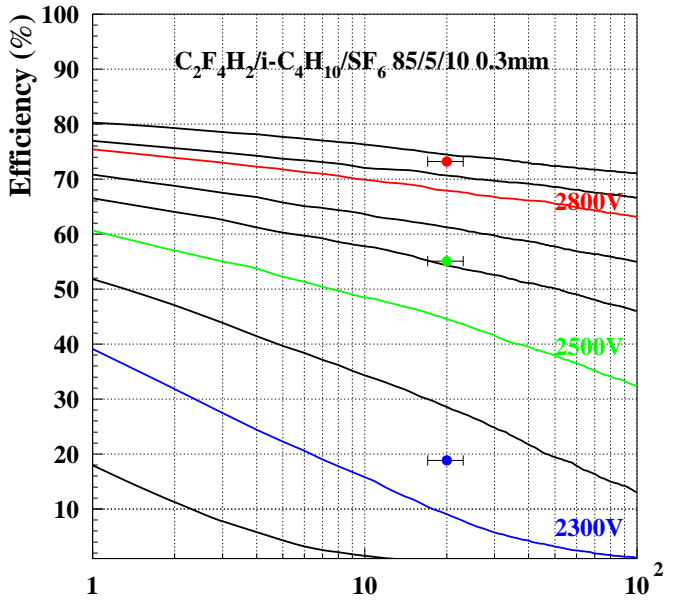

(a)

Threshold (fC)

(b)

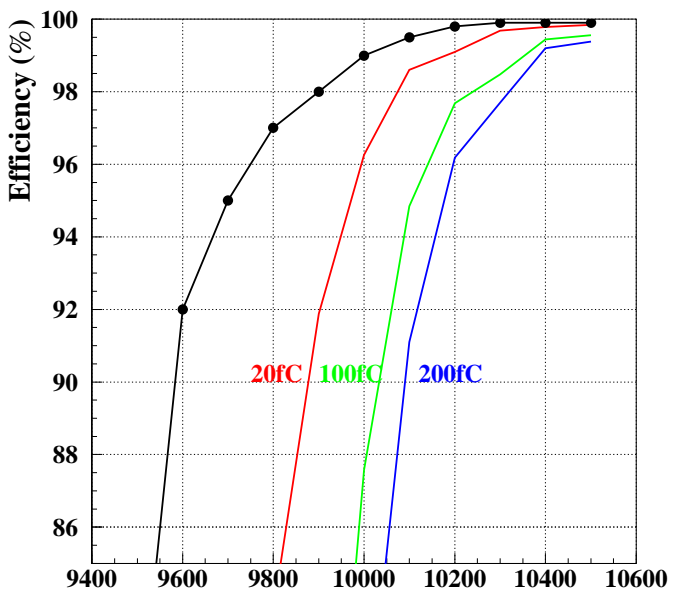

HV $(\mathbf{k V})$

Fig. 4. (a) Simulated efficiency vs. threshold for the timing RPCs for different voltages. The points correspond to measurements from [2] at the indicated voltages. (b) Simulated efficiency for trigger RPCs together with measurements (dots) from [11].

Applying a threshold to this signal gives a threshold crossing time of

$$
t(A)=\frac{1}{(\alpha-\eta) v} \ln \frac{t h r}{A}
$$

Together with an exponential pulse height fluctuation

$$
P(A)=\frac{1}{A_{a v}} e^{-\frac{A}{A_{a v}}}
$$


the time resolution is given by

$$
\sigma_{t}=\sqrt{\overline{t^{2}}-\bar{t}^{2}}=\frac{1.28255}{(\alpha-\eta) v}
$$

so we expect that the time resolution depends only on the effective Townsend coefficient and the drift velocity, not on the threshold, which is actually reproduced by the detailed Monte Carlo simulation and observed in reality. For the Trigger RPCs $(v=130 \mu \mathrm{m} / \mathrm{ns}, \alpha=10 / \mathrm{mm})$ we expect $\sigma_{t} \approx 1$ ns while for the timing $\operatorname{RPC}(v=220 \mu \mathrm{m} / \mathrm{ns}, \alpha=100 / \mathrm{mm})$ we expect $\sigma_{t} \approx 58$ ps which is close to the detailed simulation and also close to measurements. Figure 5 shows the simulated TDC spectrum for the timing RPC for $2.8 \mathrm{kV}$, and $20 \mathrm{fC}$ threshold.

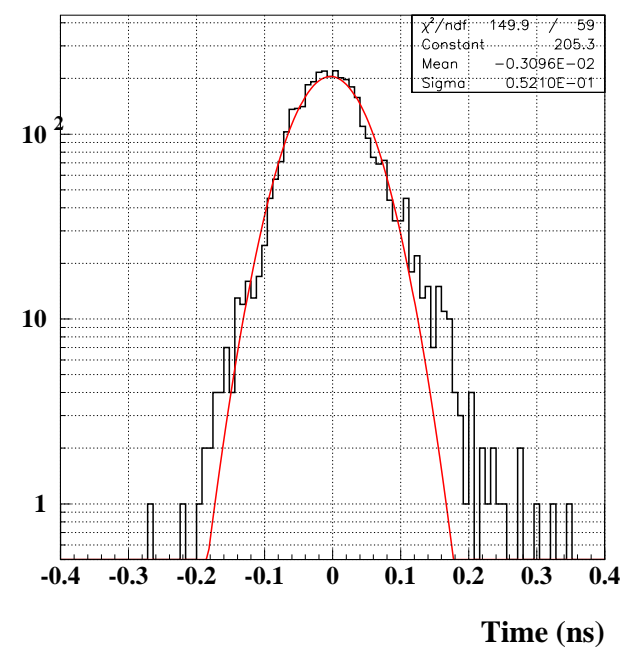

Fig. 5. TDC spectrum for a single gap timing RPC for $2.8 \mathrm{kV}$ and $20 \mathrm{fC}$ threshold giving $52 \mathrm{ps}$ time resolution. A measurement of $54 \mathrm{ps}$ for this geometry is quoted in $[7]$.

We can conclude that also the simulated time resolutions are close to the measurements.

\section{Average Charges}

The average avalanche charge is given by

$$
\bar{Q}_{t o t} \approx \frac{e_{0} n_{a v} \alpha}{\lambda(\alpha-\eta)^{2}} e^{(\alpha-\eta) d}
$$


where $\lambda$ is the average distance between clusters, $n_{0}$ is the average number of electrons per cluster, $e_{0}$ is the electron charge and all other parameters were defined above. Inserting the working point numbers quoted earlier we find average avalanche charges of $4.6 \times 10^{7} \mathrm{pC}$ and $2.2 \times 10^{5} \mathrm{pC}$ for timing and trigger RPCs which are in sharp contrast to measured numbers of 5 and $40 \mathrm{pC}$. This indicates that space charge effects must play a very prominent role in RPCs. A detailed model of this effect was presented at the same conference [12]. Implementing this effect in a crude way by simply saturating the avalanche at a certain number of electrons [13] we have to assume a saturation electron number of $1.6 \times 10^{7}$ and $2.5 \times 10^{7}$ for timing and trigger $\mathrm{RPC}$ in order to reproduce the measured charges.

\section{Conclusion}

We have simulated RPCs using 'standard' detector physics and find good agreement with measurements for efficiency and time resolution. An analytic formula for RPC time resolution also gives numbers close to experiment. The measured charges can only be explained by a very prominent space charge effect.

\section{References}

[1] R. Santonico, R. Cardarelli, Development of Resistive Plate Counters, NIM A263 (1981) et. al.

[2] P. Fonte, V. Peskov, High-resolution TOF with RPCs, Preprint LIP/00-04, (2000)

[3] Igor Smirnov, HEED, program to compute energy loss of fast particles in gases, Version 1.01, CERN

[4] S. Biagi, IMONTE, program to compute gas transport parameters

[5] S. Biagi, MAGBOLTZ, program to compute gas transport parameters, Version 2.2, CERN

[6] ATLAS Muon Spectrometer Technical Design Report, CERN-LHCC-97-22, ATLAS TDR 10, CERN 1997.

[7] P. Fonte, Development of Large Area and of Position Sensitive Timing RPCs, presentation at the 9 th Vienna Conference on Instrumentation Vienna/Austria, February 19-23, 2001

[8] R. Camarri et. al., Streamer suppression with SF6 in RPCs operated in avalanche mode, NIM A414 (1998) 317-324 
[9] Werner Legler, Die Statistik der Elektronenlawinen in elektronegativen Gasen, bei hohen Feldstaerken und bei grosser Gasverstaerkung, Z. Naturforschung. 16a, 253-261 (1961)

[10] S. Ramo, Currents induced in electron motion, PROC. IRE 27, 584 (1939)

[11] G. Aielli et. al., Performance of a large-size RPC equipped with the final ATLAS front-end electronics at X5-GIF irradiation facility, NIMA 456(2000) 77-81.

[12] Christian Lippmann, Space Charge Effects and Induced Signals in RPCs, RPC 2001, Nov. 2001, Coimbra

[13] M. Abbrescia et. al., Progress in the simulation of Resistive Plate Chambers in avalanche mode, CMS conference report CMS CR 1998/021. 